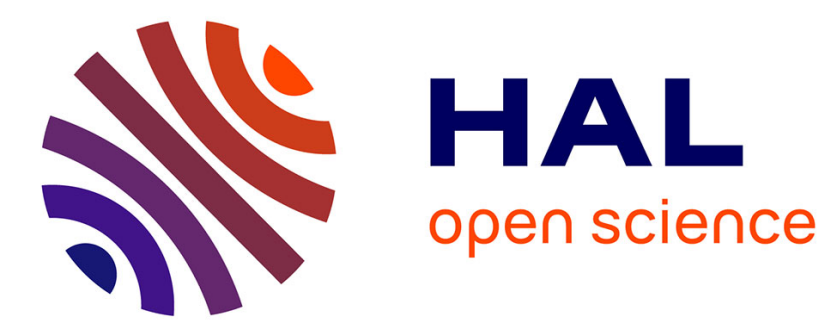

\title{
Fragments d'une économie biopolitique de la lutte contre la stigmatisation
}

Marie-Joseph Bertini

\section{To cite this version:}

Marie-Joseph Bertini. Fragments d'une économie biopolitique de la lutte contre la stigmatisation. La stigmatisation en psychiatrie et en santé mentale, ouvrage Collectif, Paris, Editions Elsevier Masson., 2010, 9782294712043. hal-03204601

\section{HAL Id: hal-03204601 \\ https://hal.univ-cotedazur.fr/hal-03204601}

Submitted on 21 Apr 2021

HAL is a multi-disciplinary open access archive for the deposit and dissemination of scientific research documents, whether they are published or not. The documents may come from teaching and research institutions in France or abroad, or from public or private research centers.
L'archive ouverte pluridisciplinaire HAL, est destinée au dépôt et à la diffusion de documents scientifiques de niveau recherche, publiés ou non, émanant des établissements d'enseignement et de recherche français ou étrangers, des laboratoires publics ou privés. 


\title{
FRAGMENTS D'UNE ECONOMIE BIO-POLITIQUE \\ DE
}

\section{LA LUTTE CONTRE LA STIGMATISATION}

\author{
Par \\ Marie-Joseph BERTINI \\ Professeure des Universités en Sciences de l'information et de la communication, \\ Directrice du Laboratoire de recherche interdisciplinaire Récits, Cultures et Sociétés
}

(LIRCES - UPR 3159)

A la lumière des travaux de Michel Foucault, de René Girard et de Pierre Bourdieu, cette étude s'interroge sur le sens et la portée des résultats de différentes enquêtes spécialisées faisant apparaître un corps de discours organisé autour d'une tripartition essentielle : dépression-maladie mentale-folie. La folie s'y dévoile comme le point aveugle, le noyau dur de la résistance des représentations sociales en santé mentale. En échappant à l'histoire et au "quadrillage" social qui contrôle la vie du sujet, la folie se renaturalise, renvoyant doublement dépressions et maladies mentales du côté du culturel. Dès lors il convient de s'interroger sur la pérennité des représentations sociales de la folie et des formes de stigmatisation qui en procèdent. Mon hypothèse est que non seulement les représentations de la folie n'évolueront que dans une faible mesure, quelques soient les moyens oeuvrant en ce sens, mais que le sens du maintien de ces représentations doit être analysé sous l'angle de sa double efficacité socio-culturelle structurante d'une part, biopolitique d'autre part.

\section{La stigmatisation, outil de socialisation}

La folie constitue en effet un agent efficace d'organisation sociale lui-même greffé sur la binarité profonde de la pensée humaine. En s'articulant sur ce double support, l'espace de la norme se déploie au sein de dispositifs techniques appropriés, dont la famille constitue le socle symbolique et idéologique. Fonction sociale mais aussi épistémologique essentielle de la folie qui définit l'absolu de la frontière hors de laquelle une entité se désagrège, l'Extérieur, le Horlà par rejet duquel s'intériorise la norme, l'impensé qui fonde toute pensée. 
Le fou est cette extériorité pure qui jamais, dans aucune culture, ne se peut inclure, frontière infranchissable et sans retour, il est cet altérité radicale qui fonde toutes les normes. L'exclusion du fou réalisée dans l'acte même de stigmatiser constitue donc un acte fondateur de la société lui permettant de fonctionner et de se réguler. La folie rejoint ici le féminin [1] dans cette extériorité dangereuse et fondatrice à partir duquel s'institue le vertige du sens. Accompagnement originel dont il conviendra d'interroger les variables cachées.

\section{1. Une construction sociale de la réalité}

L'usage du concept de représentation a fait l'objet de profonds remaniements épistémologiques ${ }^{1}$. Une société donnée existe moins en soi que dans la représentation que l'on s'en fait : anthropologues, sociopsychologues et chercheurs en sciences de l'information et de la communication sont parvenus à isoler cette idée-force indissociable de la postmodernité : les phénomènes ne valent que par les représentations que nous en avons et celleci varie sensiblement selon le contexte de leur réception. Dès lors la réalité d'un phénomène devient le produit d'un processus complexe dans lequel l'opinion, l'information et la médiatisation jouent un rôle considérable. A n'estimer que des estimations (par exemple la violence ne peut réellement s'évaluer en soi; le chercheur reste aux prises avec le sentiment de la violence qu'il essaie de mesurer et de comprendre), à fabriquer de la connaissance à partir de représentations, le chercheur en sciences humaines et sociales est confronté aux limites étroites de son propre projet. Comme si tout à coup tous ses instruments de mesure ne mesuraient qu'eux-mêmes sans rien saisir d'un réel qui leur échappe toujours puisque, telle leur ombre, ils n'existent que dans l'espace de leur propre projection.

L'une des questions centrales que pose l'économie des représentations sociales et culturelles est celle de savoir à quelles conditions la démarche même de la recherche est possible. L'analyse de discours, la sémio-pragmatique, l'interactionnisme sont autant d'outils qui aident à comprendre comment se construisent et comment évoluent nos représentations sociales, culturelles et politiques. En ce sens, les sciences de l'information et de la communication constituent un lieu privilégié à partir duquel il devient possible d'appréhender ce processus dans toute sa complexité et de tenter d'en établir des modèles. De Michel Foucault à Pierre Bourdieu, en passant par Jacques Derrida, nombreux sont ceux

\footnotetext{
${ }^{1}$ Voir les travaux de Denise Jodelet, de Serge Moscovici, de Jean-Claude Abric.
} 
qui ont travaillé à faire émerger cette idée : le monde n'est que la somme des représentations que nous en avons. Le monde comme volonté et représentation en quelque sorte, comme le marquait dès le $\mathrm{XIX}^{\circ}$ siècle le philosophe allemand Schopenhauer, selon lequel ce que nous avons coutume d'appeler phénomènes relève pour l'essentiel de nos sensations.

En ce sens nous pouvons dire que le processus connaissant relève d'une esthétique générale. Dès lors, le monde n'existe que dans son expérimentation : l'expérience du monde (aisthésis) nous tient lieu de monde, et le partage de cette expérience constitue l'ensemble du donné social et culturel. Evoluant sans cesse, tant dans ses principes que dans ses formes grâce au développement des techniques et des technologies, ce paradigme du réel est véhiculé dans des dispositifs techniques immatériels tels que les valeurs, les normes, les croyances sociales et les idéologies culturelles et politiques, comme le montrent les travaux sur la construction sociale et culturelle du Genre par exemple. L'organisation d'une mémoire et d'une intelligence collective s'articule étroitement à la prodigieuse diversité des attentes et des pratiques. La crainte de l'homogénéisation universelle des cultures est tempérée par la pluralisation et à la fragmentation des stratégies collectives et individuelles. Les modes d'appropriations des technologies de communication ouvrent l'accès à une individualité multidimensionnelle prise dans des relations de pouvoir dont elle est à la fois l'objet et le sujet ; elle augmente par là-même ce qu'Alfred Schütz appelle son "stock de connaissances disponibles" [2] et donc les interprétations potentielles de la réalité. Nos sociétés postindustrielles, marquées par l'essor technologique et l'hégémonie croissante de la culture et des médias de masse, sont aussi paradoxalement celles qui plébiscitent l'éclatement et la diversification des valeurs et des modes de vie, loin de la verticalité menaçante de "l'État universel et homogène" prophétisé par Hegel. Face à un monde où les logiques industrielles et marchandes semblent dominer, l'approche foucaldienne des représentations sociales et culturelles met en avant le rôle crucial du conflit - et donc des dynamiques contradictoires dans la construction de la réalité, notion bénéficiant d'une mise en évidence progressive au sein des sciences humaines.

Denise Jodelet définit à raison la représentation comme "une forme de connaissance socialement élaborée et partagée ayant une visée pratique et concourant à la construction d'une réalité commune à un ensemble social" [3]. L'intérêt de cette définition est de pointer et de rassembler les éléments-clefs du processus de représentation. En premier lieu la notion 
de construction commune de la réalité renvoie au partage d'un même système de signification primordial, à savoir au langage ${ }^{2}$. Dans La construction de la réalité sociale [4], John Searle poursuivant l'analyse de l'intentionnalité des états mentaux, décèle dans l'intentionnalité non plus individuelle mais collective, l'acte même de production de la société et de la culture. La théorie des actes de langage autorisent ainsi la production collective d'une réalité objective constituée de faits sociaux dont le sens déborde largement les propriétés de la réalité physique qui la sous-tend selon lui. Le langage apparaît bien ici comme le ciment du monde, son principe cohésif et organisateur ; il s'agit là d'un point particulièrement important qui met en lumière les pouvoirs du langage, ou plus exactement son aptitude à produire des normes et à les mobiliser en faveur du pouvoir de les dire, idée avec laquelle la sophistique ancienne [5] et la psychanalyse lacanienne nous ont déjà familiarisés.

En second lieu, ces représentations forment des savoirs pratiques organisant les significations relatives aux objets auxquels ils s'appliquent, et oeuvrant à une construction de la réalité conçue comme le produit des interactions communicationnelles et sociales. La fonctionnalité des représentations est ainsi mise en avant, ainsi que leur double dimension cognitive et sociale, dont Moscovici nous dit qu'elle permet d'intégrer la nouveauté et d'orienter les communications et les comportements en s'appuyant sur un ensemble de valeurs et de croyances, lui-même commandé par un principe organisateur constitué d'attitudes et de normes [6].

\section{2. La doxa ou la renaturalisation des normes}

Les représentations sociales et culturelles fonctionnent donc sur le mode de la commutation : elles transforment la réalité matérielle (individus, objets situations...) en réalité symbolique chargée de réguler les conduites, de prescrire et de proscrire, à l'intérieur d'une sémiosis générale au sein de laquelle chacun élabore les conditions de sa propre expérience. De ce fait, les représentations permettent à chaque individu de se situer par rapport aux autres d'une part et par rapport aux normes en vigueur d'autre part. Les interactions intra et inter subjectives s'avèrent médiatisées par les représentations, source des identités personnelles et sociales. Bourdieu [7] insiste sur la violence symbolique des représentations,

\footnotetext{
${ }^{2}$ Cette notion alimente un foyer théorique important, dont les diverses expressions (notamment anglo-saxonnes) sont rassemblées autour du nom générique de "Common Knowledge".
} 
autrement dit sur leur caractère dominateur et arbitraire. Cette violence s'exerce à travers des procédés visant à naturaliser les normes, à rendre invisible leur relativité et leur culturalité historiquement déterminée, donc ouverte à la remise en cause critique. Elle est "un travail historique de déhistoricisation" [8] et culmine dans la reconnaissance individuelle et collective de la légitimité des représentations. En s'y conformant, chacun prend la place qui lui est attribuée dans le concert social et renonce du même coup à son pouvoir de déconstruction et de transformation.

Ce que nous choisirons d'appeler avec lui la doxa, désigne donc un ensemble d'opinions, de valeurs, de croyances, de normes, établies comme allant de soi et constituant un arbitraire culturel propre à une société donnée à un moment donné de son histoire. La finalité de la doxa entendue comme système symbolique consiste à maintenir l'état fluctuant du réseau de relations de force, à pérenniser la reproduction dynamique de la structure sociale. C'est pourquoi Bourdieu nous rappelle que notre première expérience du monde est celle de la doxa qui façonne les logiques de la conformité et détermine ainsi les formes de l'objectivité [9]. La doxa constitue donc la matrice des procédés de renaturalisation du culturel. Elle est le centre nerveux à partir duquel rayonne les modes d'organisation sociocognitifs de la réalité.

C'est la raison pour laquelle les sciences de l'information et de la communication offrent un angle de vision particulièrement révélateur du fonctionnement de la doxa. Elles permettent de mettre en évidence l'adossement de cette dernière aux stratégies de communication et d'information et à leurs instruments techniques, médiatiques et discursifs. Les modes de circulation de la doxa dans le tissu social sont indissociables des technologies de communication et des systèmes médiatiques qui en dérivent : en ce sens, elles constituent des technologies du pouvoir dont il nous faut chercher à identifier les aspects et à repérer les effets contradictoires et complémentaires. Les stratégies discursives figurent en bonne place parmi ces technologies de communication/pouvoir. Rappelons que Michel Foucault définit le discours comme l'ensemble des significations contraintes et contraignantes (normatives), qui circulent à travers les rapports sociaux et qu'il faut analyser comme un champ stratégique en modification permanente, un lieu d'affrontement où "prendre position" s'entend comme le mouvement d'une armée occupant les points-clefs du terrain. Le pouvoir est d'abord et avant tout pouvoir d'imposer des significations, d'opérer des partages entre le 
monde de la signification et celui de l'insignifiance, entre langage et gesticulation, raison et délire, pondération et hystérie. Même si les frontières bougent, évoluent, se transforment, le pouvoir est toujours consubstantiel à la dernière frontière en date, soudée à elle.

Dans son ouvrage publié en 1982, Ce que parler veut dire [10], Pierre Bourdieu met en avant la dimension de signification autoritaire des discours, moins destinés à être compris et interprétés qu'à entraîner la croyance et l'obéissance. Il décrit le discours comme un croisement entre un habitus linguistique - une compétence technique et sociale - et un marché linguistique, un système de règles fixant les modes d'évaluation sociale et orientant par avance toute production linguistique. De même, dans Langage et pouvoir symbolique [11], l'auteur analyse le pouvoir symbolique du langage comme pouvoir de faire advenir le donné dans l'énonciation, de montrer et de cacher, de modifier la vision de la réalité et partant, les modes d'action sur elle. Le travail du chercheur consiste dès lors à analyser attentivement les discours qui mettent le monde en ordre et portent en eux la trace du marché dont ils sont le produit, et dont ils contribuent à entretenir les artefacts. Comprendre les mécanismes de fonctionnement de la doxa constitue donc l'étape indispensable d'un type de recherches à visée transformatrice. C'est la raison pour laquelle il est particulièrement intéressant d'étudier l'un de ses modes d'expression majeur que constituent la folie et la maladie mentale. Il s'agit d'analyser de manière approfondie et détaillée d'une part la façon dont les discours fabriquent de la norme en cet endroit stratégiquement situé, et d'autre part d'étudier les effets de ces discours au sein de la construction sociale et politique de la réalité.

\section{3. Une sociodicée de la santé mentale}

Le groupe de recherche sur les représentations de la santé mentale s'est constitué au début de l'année 2003 autour de médecins psychiatres et chefs de service de psychiatrie en établissements hospitalo-universitaires, de psychologues cliniciens et psychanalystes, d'anthropologues, d'une philosophe, d'un théologien, de chercheurs en sciences de l'information et de la communication. L'équipe, fortement structurée autour d'un noyau de six chercheurs permanents et de trois doctorants en SIC, bénéficie d'éclairages théoriques diversifiés appuyés sur une double pratique médico-hospitalière et universitaire. Elle travaille plus particulièrement sur les discours - tenus par un public de non spécialistes portant sur la folie et sur la maladie mentale, ainsi que sur les représentations sociales et 
culturelles qui les sous-tendent. Elle vise donc d'une part à connaître les opinions, les ressentis, les idées, les préjugés du grand public par rapport aux principaux termes utilisés couramment pour désigner les personnes atteintes de troubles mentaux (dépressifs, malades mentaux, fous). D'autre part à évaluer ses réactions à l'égard de ces derniers, mais aussi à l'égard des différents modes de soins disponibles et utilisés.

Prenant appui sur l'essor de la mondialisation de la santé, ses recherches s'effectuent notamment autour d'une enquête majeure intitulée "La santé mentale en population générale : Images et réalités", sous la responsabilité de l'Organisation mondiale de la Santé (OMS). Initiée par l'OMS en France et dans l'Océan indien cette enquête vise à mieux connaître et comprendre les représentations collectives de la santé mentale selon un double axe local et global. Cette recherche implique directement sur le terrain les personnels de santé, dans certaines régions particulièrement sensibles sur le plan épidémiologique. L'intérêt tout particulier de cette recherche, outre son caractère interculturel et interethnique, est de marier de manière originale enquête épidémiologique et enquête anthropologique, menée en population générale et non parmi les malades diagnostiqués par les systèmes de soins. Cette étude mondiale menée durant plusieurs années sur 45 sites français, dont trois sites hors hexagone (Guadeloupe, Martinique, Réunion), sur des sites étrangers notamment dans l'océan indien (Ile Maurice, Comores, Madagascar) et autour du bassin méditerranéen ( Grèce, Espagne, Italie, Algérie, Tunisie) a mobilisé des moyens considérables. En France métropolitaine et hors métropole, un échantillon de 40.000 personnes sélectionnées selon la méthode des quotas ${ }^{3}$, a ainsi été mis au point et soumis à une enquête par questionnaire permettant à des personnels compétents (infirmiers et élèves-infirmiers) spécialement formés à cet effet, de recueillir des données au cours d'entretiens en face à face.

L'étude se centre donc sur ces trois mots appartenant au vocabulaire de la santé mentale : fou, malade mental, dépressif. Il s'agit en particulier de cerner la perception de ces trois phénomènes dans l'opinion, en faisant associer à ces termes dix-huit comportements ou conduites reconnues comme illustratives de troubles mentaux (tenir un discours dépourvu de sens, être bizarre, négligé,...). En outre, les personnes interrogées doivent préciser si ce comportement leur apparaît normal ou anormal, dangereux ou inoffensif. Le questionnaire

\footnotetext{
${ }^{3}$ La structure de la population sur laquelle portait l'enquête a ainsi été calée sur la structure par sexe, âge, et catégorie socio-professionnelle de la population générale de la zone géographique enquêtée.
} 
comprend une cinquantaine de questions ouvertes, semi-ouvertes et fermées qui permettent d'explorer les représentations liées à trois phénomènes : la folie, la maladie mentale et la dépression, mais aussi les différents types d'aide potentiels et les modalités de soin envisageables, la tolérance de la société et le rôle de la famille. Pour chaque situation ou comportement proposé lors de l'enquête, la personne interrogée doit préciser si ce dernier relève de l'un des trois troubles cliniques précités.

L'étude effectué sur le site de Nice - site retenu par l'OMS - a été menée auprès de 900 personnes volontaires constituant un échantillon représentatif d'une population résidant dans certains quartiers et cantons (soit 80.000 habitants) interrogé entre le 30 mai et le 7 juin 2003 par une soixantaine d'enquêteurs. Trois questions ouvertes étaient posées en première intention : selon vous, qu'est-ce qu'un fou - un malade mental - un dépressif ? Les réponses spontanées des personnes interrogées ont été notées mot à mot par les enquêteurs et les réponses ont été analysées au moyen du logiciel d'analyse de données textuelles Alceste ${ }^{4}$. Il en ressort deux éléments importants : une cohésion des représentations sociales sur l'ensemble du site concerné, mais aussi pour l'ensemble des sites français concernés. Le profil des réponses en effet ne montre pas de différences significatives par rapport à l'analyse réalisée pour l'ensemble des sites français ayant participé à l'enquête.

\section{4. L'organisation discursive de la dépression}

Le résultat le plus remarquable concerne les différences fortes établies par les personnes interrogées entre dépression d'un côté et maladie mentale et folie de l'autre. Bien individualisée par rapport aux deux autres concepts jugés plus flous, la dépression apparaît comme une notion familière marquée par un vocabulaire spécifique. Le dépressif est envisagé au sein des représentations étudiées dans des termes voisins d'un ouvrage de psychiatrie; la tristesse de l'humeur, le déclin de l'énergie et la fatigabilité, la diminution de l'intérêt et du plaisir, le manque de contrôle des émotions, le ralentissement intellectuel, y apparaissent comme constitutifs du syndrome dépressif. Les causes des symptômes - ce point est important pour la mise en perspective de l'enquête - sont attribuées au milieu extérieur, à l'environnement social en particulier. Les représentations cernent le dépressif sur le mode victimaire; malmené par les événements de la vie, sa dépression est perçue comme

\footnotetext{
${ }^{4}$ Analyse lexicale par contexte de segment de texte, Alceste travaille sur trois unités de signification : les mots, les unités de contexte, le corpus (texte global).
} 
le produit de déceptions, de chocs et de conflits. Le terme dépressif est renvoyé à des troubles considérés comme relativement normaux $(75 \%$ des personnes déclarent en connaître au moins un dans leur entourage proche). En outre, la très grande majorité considère que celui-ci peut guérir de manière complète. Ces considérations expliquent la recommandation de prises en charge thérapeutiques en milieu externe et non hospitalier.

Les représentations sociales et culturelles font donc ici apparaître la dépression comme le trouble mental le mieux accepté au sein de la société contemporaine, et par conséquent le plus facilement avouable. En ce sens il semble se détacher du tableau clinique classique dont il ne constitue plus l'un des premiers niveaux de l'échelle des maladies mentales, mais s'inscrit dans le répertoire des troubles de la vie quotidienne auxquels les effets de la médiatisation (télévision, radio et presse écrite pour l'essentiel) préparent de mieux en mieux un public de plus en plus informé. De ce résultat, on pourrait être tenté de conclure que l'information peut influer directement, à court et à moyen terme, sur les représentations de la santé mentale; nous verrons cependant ultérieurement qu'il n'en est rien.

\section{5. L'organisation discursive de la maladie mentale}

Le terme "malade mental" laisse apparaître une grande ambivalence entre origine somatique et origine psychologique, comme le montrent les expressions faisant référence aux deux champs sémantiques. Cette confusion est attestée par les unités de contexte élémentaires recueillies; la folie est évacuée au profit de l'idée de "pathologie mentale", de "troubles psychiatriques", relativement bien identifiés autour de la schizophrénie et de la psychose. Très liée dans l'esprit des enquêtés à l'hopital psychiatrique, la maladie mentale est renvoyée du côté des transgressions sociales associées à la violence sexuelle, intime et familiale, à la dangerosité, au délire et aux hallucinations, sans toutefois déboucher sur le meurtre. La maladie mentale appelle pour la population générale des soins prolongés en milieu hospitalier; cette dernière évoque spontanément des conséquences lourdes notamment sur l'existence du malade et sur son entourage amical et familial. Si, par certains traits dégagés, les représentations de la maladie mentale et celles de la folie voisinent, elles n'en demeurent pas moins claires sur un point précis : le malade mental n'est pas un fou; il souffre d'une "maladie du cerveau" sérieuse, certes, mais qui est susceptible d'être soignée par des soins médicaux appropriés, pouvant mener à la guérison, même s'il faut pour cela aller contre la volonté du malade. Cette guérison est cependant jugée inhérente à des traitements longs et 
lourds, à la fois médicamenteux et psychothérapeutiques, nécessitant des contraintes hospitalières sporadiques. Considéré comme "exclu de la société" et "exclu de son travail", le malade mental, comme le dépressif, n'est pas jugé exclu de sa famille, un peu moins de la moitié des personnes interrogées se déclarant prête à le soigner pour une part au domicile familial, même si l'accueil et les soins à domicile sont ressentis comme une charge pesante pour les proches.

\section{6. L'organisation discursive de la folie}

Les unités de contexte élémentaires mises à jour par l'enquête ont révélé à cet endroit les éléments qui paraissent les plus significatifs des formes du discours sur la santé mentale. Jugé totalement irresponsable, ne se rendant aucunement compte de ce qu'il fait, le fou est assimilé à une personne dont le comportement "bizarre" et "différent" ne correspond pas à une norme et l'extrait de la réalité commune. L'absence de contrôle de soi est reliée à l'absence de conscience de soi permanente, faisant obstacle à l'analyse et à l'évaluation de la portée de ses actes. Le fou est considéré comme un individu ayant perdu le sens du rationnel et réagissant "hors des normes et des codes de la société". Comme on peut le voir, on atteint ici pour un public de non spécialistes, un degré très élevé des troubles cliniques. Jugé incurable à une très grande majorité $(90 \%)$ et donc ne requérant aucun soin thérapeutique considéré comme inutile, le fou fait partie d'un monde étranger et incompréhensible, incapable de toute adaptation à la vie normale, et ce, de manière irrémédiable. Particulièrement violentes et dangereuses, ses conduites appellent l'enfermement asilaire prolongé voire définitif. Le caractère imprévisible et incompréhensible de ces dernières est mis en avant pour justifier cette réclusion. Une partie non négligable de la population (2 à $10 \%$ ) va jusqu'à refuser à la folie le statut de maladie, relié dans ses présupposés à l'efficacité potentielle d'un traitement médical. Il résulte de l'ensemble une vision de la folie mystérieuse dont les origines sont floues et mal identifiées. Une vision désespérée également, comme un voyage sans retour et sans compagnon possible qui renvoie à une mort sociale inéluctable. Indissociable de la notion d'exclusion et de refus d'intégration, la folie inspire des craintes profondes et généralisées, modulées selon l'éthos culturel d'appartenance, mais manifestes au sein de la totalité des espaces culturels parcourus par l'enquête. 


\section{7. A quoi sert la folie ?}

Une telle enquête, marquée par une amplitude géographique et culturelle considérable, constitue un matériau d'une grande richesse mis à la disposition du chercheur en sciences humaines et en sciences sociales. Elle permet en particulier d'étudier de très près les discours portant sur la santé mentale en France et dans de nombreux pays du monde, ainsi que les représentations sociales et culturelles qui les sous-tendent. Le travail remarquable de Michel Foucault sur la folie à l'âge classique s'est effectué sur des représentations mobilisées par des discours d'archive. Ici, le chercheur a à faire avec une parole vivante qui réactualise et recadre le donné historique. On n'ose imaginer l'opportunité qu'un tel matériau aurait constitué pour Foucault, et c'est d'ailleurs dans cette perspective qu'est rédigé cet article, c'est-à-dire en essayant de saisir la manière dont ces discours spécifiques prennent place et influence au coeur de la doxa de nos sociétés contemporaines. La question centrale sera donc à cet endroit celle de savoir quel rôle spécifique joue la folie et ses discours d'accompagnement dans notre société. En d'autres termes : à quoi, et à qui, sert la folie ?

Les enjeux individuels et sociaux de la santé mentale sont révélateurs de significations essentielles qui nervurent une société. Celles-ci sont matérialisées par des dispositifs matériels nombreux tels que l'hygiène, les soins, la libre circulation, les techniques disciplinaires, qui se renversent aussitôt dans des dispositifs symboliques (en l'occurrence, la salubrité, la santé, la liberté, le contrôle de soi) d'une part. D'autre part ces dispositifs établissent une continuité logique entre corps individuel et corps social ; ils constituent donc les éléments centraux d'une physiologie et d'une psychologie sociale dont les formes sont évolutives en dépit de leur grande stabilité dans le temps. Les interprétations des pathologies mentales apparaissent ainsi articulées à l'histoire des mentalités et révèlent les conflits et les tensions auxquelles ces interprétations ont donné lieu.

Ces pathologies présentent la particularité de ne pas pouvoir être associées à des comportements dits "à risque" permettant de déployer des systèmes de prévention et de protection ; elles sont au contraire le produit d'interactions complexes entre processus biologiques, processus sociobiologiques (génétiques et héréditaires en particulier) et processus psychiques et sociaux. Cette complexité appelle des approches et des analyses 
médicales mais aussi non médicales, particulièrement bienvenues quand elles relèvent des sciences humaines, et sollicitées par les professionnels de santé eux-mêmes. Ces dernières présentent également l'avantage non négligeable de mettre en évidence les mécanismes de médiation médicale croissante des interactions sociales que dénonçait déjà Michel Foucault et qu'il attribuait en particulier à l'institution d' une fonction psy chargée selon lui de généraliser et de multiplier les modes de contrôle sur les individus et les groupes.

Toutefois, les discours sur la santé mentale s'avèrent marqués par deux mouvements complémentaires articulés autour d'une évolution sensible d'un côté, d'une remarquable stabilité de l'autre. Les évolutions tiennent aux modifications de la nature et de la fonction du savoir et du pouvoir médical en matière de santé mentale. Les médias jouent notamment aujourd'hui un rôle d'information et d'éducation qui a fortement contribué à modifier l'image de la dépression et de la maladie mentale. La fonction psy se trouve ainsi débordée et englobée par une fonction d'information et de communication qui relativise les pouvoirs de la première, en la mettant en scène et en l'instrumentalisant au profit de dispositifs médiatiques avides des taux d'audience croissants suscités par les problèmes posés par la santé mentale.

Autrefois tenues au secret, dissimulées derrière les hauts murs de l'asile, les pathologies mentales sont conviées à s'exposer, à se donner à voir jusque dans leurs détails les plus singuliers. Fait remarquablement nouveau, le discours sur la maladie mentale se double depuis du discours $d u$ malade mental jusqu'ici réduit au silence et à l'insignifiance. Les dispositifs médiatiques concourent ainsi à cerner et à isoler la part d'humanité qui perdure malgré les attaques de la maladie : par-là, ils contribuent à réintégrer le malade mental dans la société, familiarisée qu'elle devient avec des troubles aussi étranges autrefois que les TOC (troubles obsessionnels compulsifs) l'autisme, l'hyperactivité, la maniacodépression, la paranoïa, ou la schizophrénie.

L'autre évolution de la fonction psy tient à la contradiction qui s'établit depuis une trentaine d'années ${ }^{5}$ environ, entre les ressentis des professionnels de la santé et le grand public, les premiers s'avouant impuissants à contenter les seconds, c'est-à-dire à protéger la

\footnotetext{
${ }^{5}$ En fait depuis la mise en place de la psychiatrie dite de secteur, marquée par l'hospitalisation en milieu ouvert, mais aussi par l'hospitalisation de jour et les soins partagés entre domicile, hôpitaux et cliniques.
} 
société des atteintes potentielles perpétrées par les malades mentaux. La psychiatrie n'a cessé ces dernières années de tenter de décloisonner les pathologies mentales, de renvoyer les individus qui en souffrent dans la société. Les raisons économiques qui sont avancées masquent mal la volonté de refuser la dimension pénitentiaire autrefois indissociable des troubles de la santé mentale. En 1881, le romancier Machado publia L'Aliéniste [12], fable acide sur la folie et l'idée que l'enfermement produit la folie et non l'inverse : un aliéniste fonde un asile "la maison verte" et y enferme tous ceux qui ont des comportements anormaux; de plus en plus de gens y sont enfermés ; bientôt la presque totalité de la ville y est et l'aliéniste finit par se demander si ceux qui sont restés dehors ne sont pas les plus dangereux.

Accusée de renvoyer abruptement les malades dans la société (et en particulier les fous), de s'en débarrasser sur elle, la psychiatrie occidentale contemporaine a rompu avec son ancêtre immédiate ; elle ne se sert plus de la peur du fou pour maintenir son pourvoir, mais au contraire elle lutte contre cette peur et travaille à réhabiliter les fous aux yeux du grand public. Que s'est-il passé entre les deux ? Pourquoi un tel basculement, un tel renversement? Michel Foucault y lirait sans doute une extension de la fonction psy à la société tout entière, montrant par là-même à quel point celle-ci a intégré et intériorisé les structures normalisantes, comme nous le verrons. D'abord traitée par le médecin, la maladie mentale fait l'objet de traitements télévisuels qui instituent de nouveaux modes d'appréhension et de définition ; elle devient ainsi un sujet de société. Psychiatrisation de la société et socialisation de la psychiatrie s'accordent probablement ici plus qu'il n'y paraît au premier abord. Notre analyse s'appuie sur une différenciation essentielle qui s'est trouvée confortée et validée par les résultats de l'enquête générale.

\section{8. Une différence fondatrice}

La tripartition établie autour des trois catégories de dépression, de maladie mentale et de folie génère trois discours étroitement articulés les uns aux autres. Notons au passage la différence des verbes utilisés pour les désigner : le fou est fou, le malade $a$ une maladie mentale, le déprimé fait une dépression : de l'être à l'avoir en passant par le faire, les glissements sémantiques s'avèrent révélateurs d'une démarche globale. Le discours sur la folie et la stigmatisation [13] qu'il exprime sont particulièrement intéressants. L'un des 
principaux résultats de l'enquête en effet, a été de mettre en évidence l'absence totale d'évolution des discours (et des représentations qui leur sont sous-jacentes) concernant la folie, ce quel que soit le sexe, l'âge, la catégorie socio-professionnelle, le niveau d'études ou l'éthos culturel auquel appartiennent les personnes interrogées. Autrement dit, les évolutions mentales et sociales ont glissé sur la folie sans jamais en altérer les significations primordiales. A l'historicité revendiquée de la dépression et de la maladie mentale, passagères et liées aux tumultes de la vie, fait face l'anhistoricité de la folie. La dimension culturelle des premières se heurte à la prétendue naturalité de la folie. Du discours sur la folie émerge les structures d'une sociodicée au sein de laquelle l'exclusion du fou joue un rôle fondateur, semblable au pomerium de la Rome antique, enceinte sacrée d'une ville, et plus spécialement limite de la cité romaine que Romulus Rex - étymologiquement tireur de trait - traça d'un sillon lors de la fondation de Rome. "Pas de cultures sans clôtures", écrit Daniel Bougnoux [14].

Le fou y apparaît en effet clairement comme un Horlà, terme que l'on peut écrire en suivant la graphie de Maupassant [15] ou bien ainsi : Hors-là. Il est unanimement décrit comme étant hors de la norme, hors de la conscience, hors de la réalité, hors de la famille, hors de la société, hors de la raison, hors du monde et surtout hors de lui-même. Le fou "n'a pas de psychologie" insistent certaines personnes interrogées, indiquant par là qu'il ne possède pas d'intériorité, qu'il est une pure extériorité. Et c'est bien ainsi qu'il nous est utile. La folie constitue le noyau dur des pathologies mentales; elle est irréductible en ce sens qu'elle ne se laisse pas réduire, ramener à, parce qu'elle est elle-même un principe normatif essentiel sur lequel se fondent les rapports qui dépendent de lui.

Notre analyse des discours sur la folie nous conduit donc à attribuer à cette dernière une fonction épistémique et sociale. Epistémique en premier lieu, parce qu'elle constitue le point aveugle de tous nos systèmes de signification et de nos savoirs : elle est l'impensé qui gît, interrogé, au fondement la pensée, ce non-signifiant qui fonde la signifiance, cette altérité radicale sur laquelle médite Maurice Blanchot dans Faux Pas [16]. La folie matérialise la frontière qui sépare la raison de son autre, car que serait une raison qui ne se peut inverser dans la déraison ? Il y a deux sortes de frontières : les frontières poreuses et franchissables derrière lesquelles se trouve momentanément la dépression et la maladie 
mentale. Celles-ci fonctionnent à la manière des membranes qui protègent mais négocient subtilement en même temps les échanges de l'intérieur vers l'extérieur.

Les frontières de la folie sont infranchissables dans la mesure où elle est cette pure Extériorité qui ne se peut jamais et nulle part s'inclure, dans quelque culture que ce soit, et à quelque époque que ce soit. Ce dernier point nous conduit à la fonction sociale de la folie qui se présente et s'impose comme un agent d'organisation sociale permettant d'une part de justifier les dispositifs de contrôle et de coercition des individus et des groupes : ce qui est jugé déviant ou insupportable est assimilé à la folie, et les asiles ont longtemps constitué des lieux de relégation des fous mais aussi de toutes les formes de déviations sociales comme le révélèrent les travaux de Michel Foucault sur les archives de la Salpétrière à Paris.

C'est la folie, d'autre part, qui préside aux opérations d'inclusion-exclusion en tant qu'elle institue la frontière fondamentale sur laquelle se fonde le principe même du dehors/dedans, exclu/admis, accepté/rejeté, principe qui organise et régule l'ensemble des interactions sociales. Le rejet du fou permet d'opérer la synthèse sociale. Le sociologue Georges Simmel [17] nous dit que toute relation sociale est le résultat d'un processus de séparation et de liaison. C'est pourquoi dans son ouvrage Stigmate, Goffman affirme que le processus de stigmatisation n'est qu'un cas particulier des interactions sociales en général, ajoutant que tout le monde est stigmatisé sauf les hommes jeunes, blancs, hétérosexuels, urbains, pères de famille, ayant fait des études supérieures, en bonne santé et n'ayant pas de problèmes de poids.

La violence du rejet exercé à l'encontre de la folie, nie à celui qui en porte les stigmates toute part d'humanité et renvoie son discours (à la différence de celui des dépressifs et des malades mentaux) vers le silence de l'insignifiance primordiale. La théorie de la victime émissaire de René Girard est utile ici pour comprendre ce que cette violence sacrificielle possède d'essentiel pour la société : l'exclusion définitive de la folie permet à la société de fonctionner et de se réguler.

Son fondement n'est pas moral, mais pragmatique : il "ne doit donc pas se définir en termes de culpabilité et d'innocence. Il n'y a rien à "expier". La société cherche à détourner vers une victime relativement indifférente, une victime "sacrifiable", une violence qui risque 
de frapper ses propres membres, ceux qu'elle entend à tout prix protéger"6 [18]. Dans son important travail sur les représentations sociales de la folie, Denise Jodelet décrit les modes de séparation d'une exceptionnelle rigueur établis entre "bredins" (le nom local des fous) et "civils". L'interdiction des mariages notamment, préserve ainsi la hiérarchie sociale mais surtout conjure le risque majeur menaçant l'identité collective : l'assimilation et l'indifférenciation "bredin-civil." L'intégrité du corps social ne peut donc être maintenue qu'à la condition de pratiquer une exclusion sans exception de tout individu s'orientant vers une tentative d'assimilation. Les études de Jodelet font ainsi apparaître l'importance vitale des pratiques de séparation pour la société, organisées autour de tabous de contact destinés à éviter toute pollution ou contamination ${ }^{7}$. La société semble ainsi assurer la pérennisation de ses représentations de la folie parce qu'elle tient à se protéger d'un péril majeur. Dans l'espace de la langue se déploie le danger de se tenir au sortir de la consistance du monde, à l'orée de son inconsistance, là où l'individu et le groupe manquent de s'abîmer dans le désorganisé. Le devenir-fou que fait miroiter le discours fonctionne alors comme un révélateur de l'angoisse sociale archaïque, c'est-à-dire première : le retour à l'indifférencié.

D'où les résistances très fortes qui sont mobilisées pour faire obstacle à toute tentative d'intégration, y compris celles qui viennent des psychiatres eux-mêmes. Les discours du grand public réagissant à l'effort de certains professionnels pour essayer de faire évoluer les représentations de la folie, sont révélateurs à cet égard. Les médias se font le relais de propos mettant en avant leur traîtrise et leur irresponsabilité supposées, les soupçonnant de ne pas être eux-mêmes tout à fait sains d'esprit, par les effets d'une contamination subordonnée à une proximité jugée trop grande ${ }^{8}$. Cette proximité du soignant et du soigné est vécue par le grand public comme moins protectrice, et partant, moins légitime que la distance infranchissable établie autrefois entre l'aliéniste et le malade. L'autorité publique est donc conviée à prendre toute sa place dans ce dialogue impossible. Sensible aux arguments des spécialistes, mais responsable de l'ordre public, le politique navigue depuis plusieurs années entre écoute bienveillante et fermeté, multipliant les expertises et les "plans santé mentale", avide de solutions rendues impossibles en raison

\footnotetext{
${ }^{6}[18]$, page 13 .

${ }^{7}$ Ainsi, en vertu d'une règle implicite mais imprescriptible, leur linge et les ustensiles de leur vaisselle étaient lavés à part et conservés à l'écart de ceux appartenant à la famille d'accueil.

${ }^{8}$ Voir à cet effet les dossiers consacrés par les médias de presse écrite à la santé mentale et à ses représentations, lors de récentes et moins récentes affaires criminelles mettant en cause des personnes atteintes de pathologies mentales.
} 
même des fonctions épistémique et sociale de la folie. Fondatrice de l'ordre social, l'éviction de la folie procède d'un mouvement pérenne. De ses modes d'exclusion symbolique, rituel et pratique, dépend l'équilibre de sociétés qui dépensent une grande énergie dans ces opérations de contention, d'extraction et de marginalisation.

La Folie continue d'émerger comme un opérateur de socialisation qui permet aux individus et aux groupes de se constituer contre elle, en opposition à elle. En nous obligeant à affronter la pérennité des représentations sociale de la folie et leur caractère profondément transculturel, les analyses des plus récents discours produits par nos sociétés contemporaines nous permettent de comprendre combien la Folie, Dehors et Désordre mêlés, nous met en rapport avec l'absolument autre que décrivait Maurice Blanchot: Altérité radicale et civilisatrice qui renvoie brutalement la stigmatisation qui procède d'elle à la difficile réalité d'un passé qui ne change pas.

\section{Stigmatiser la stigmatisation : pour une socio-analyse de la stigmatisation}

Dans leur rapport de mission "De la psychiatrie vers la santé mentale" [19] présenté en Juillet 2001, Eric Piel et Jean-Luc Roelandt analysent avec finesse la nature du glissement conceptuel et pratique qui s'opère à l'aube des années 2000 en direction de la notion de "santé mentale". Ce faisant, ils désignent la lutte contre la stigmatisation comme le point d'orgue de la psychiatrie de secteurs, elle-même considérée comme l'ultime étape avant la réintroduction totale du malade mental dans la société. "Pendant deux siècles", écrivent-ils en introduction à leur rapport, "les médecins des âmes se sont enfermés avec leurs "fous" dans les asiles d'aliénés. Pour les protéger bien sûr et pour "protéger" la société aussi. Mais l'enfer était pavé, ici comme ailleurs, des meilleures intentions. Il s'agit maintenant de rendre possible le dernier acte de la fin de cet exil des "fous" et de l'enfermement, de permettre l'aboutissement de la politique de sectorisation et son ouverture à la population, à la société". Parmi les principes généraux énoncés dans ce rapport, on peut lire l'affirmation suivante : "Il faut aller au terme de la politique de sectorisation, pensée en 1945, et fermer les lieux d'exclusion médicaux et sociaux". Un programme aussi ambitieux doit se doter d'instruments concrets de changement des mentalités. C'est pourquoi, un peu plus loin, les deux auteurs avancent : "La lutte contre la stigmatisation dont sont victimes les personnes souffrant de troubles mentaux est indispensable. La population doit être sensibilisée, afin de 
modifier les préjugés de dangerosité, d'incompréhension et d'incurabilité des troubles mentaux".

\section{1. Le concept de santé mentale : une axiomatique générale}

Le grand mérite de ce rapport est de poser les termes de l'équation à laquelle nos sociétés sont confrontées aujourd'hui. La notion de santé mentale a l'immense mérite de repositionner la maladie mentale au sein de la cartographie générale des maladies humaines et par conséquent de l'extraire du registre pathologique spécifique qui était le sien jusqu'alors. Elle ne relève plus de mesures particulières telles que celles qui conduisaient à isoler le malade mental de l'ensemble du corps social. En ce sens, cette notion de santé mentale est elle-même le produit de l'évolutions des méthodes de soins alternatives initiées dès les années 70, en relation avec l'intense activité philosophique et intellectuelle qui s'était emparée de la problématique du fou et de la folie. Michel Foucault, Gilles Deleuze, Félix Guattari, psychiatres et anti-psychiatres, ont nourri les débats passionnés de cette époque qui ont permis de repenser notre rapport à la folie et, au-delà de celle-ci, à la raison et à ses modes de définition. "On ne peut pas penser n'importe quoi, n'importe quand" avait coutume de dire Foucault, autrement dit notre socio-culture française s'est préparée lentement à partir de là à accueillir les évolutions profondes qui durant les trente années suivantes allaient la conduire à penser les nouveaux modes d'intégration des malades mentaux au sein de la Cité. Intégrative, la notion de santé mentale est aussi désintégrative au sens où elle procède d'une déconstruction des représentations des pathologies mentales. Ce faisant, elle transfère vers les spécialistes de ces pathologies, médecins-psychiatres, psychologues, psychanalystes,... la charge de participer activement à cette déconstruction ce qui constitue un premier point d'achoppement sur lequel nous reviendrons.

Cette notion de santé mentale présente un autre intérêt : par elle, ce n'est plus le concept de maladie qui est central mais celui de santé. Autrement dit, elle fait basculer la santé du côté d'une axiomatique générale, c'est-à-dire d'un ensemble de propositions admises sans démonstration. Ce qui n'est pas sans effet sur la suite car cela signifie que la santé est pensée comme un point de départ et non comme un point d'arrivée, autrement dit comme une norme à partir de laquelle il faut établir les déviations possibles, les anticiper et les corriger. Intégrative, déconstructive et normative, la notion de santé mentale présente 
toutes les caractéristiques qui président à l'établissement d'une structure paradoxale que nous nous proposons d'interroger ici. Sur son site Internet, l'OMS (Organisation Mondiale de la Santé) présente ainsi cette notion : "La santé mentale fait l'objet d'un large éventail d'activités qui relèvent directement ou indirectement du "bien-être", tel qu'il figure dans la définition de la santé établie par l'OMS comme étant "un état de complet bien-être physique, mental et social, qui ne consiste pas seulement en une absence de maladie ou d'infirmité. La santé mentale englobe la promotion du bien-être, la prévention des troubles mentaux, le traitement et la réadaptation des personnes atteintes de ces troubles". La médecine se livre ici aux mêmes aspirations que l'économie qui, sous la plume d'économistes célèbres, tels que le prix Nobel d'économie Joseph Stieglitz, se propose de remplacer le calcul du PIB (produit intérieur brut) par celui du BIB (bien-être brut). Dans les deux cas, comment le mesurer?

La question centrale devient donc celle des indicateurs, indicateurs de santé et de richesse qui sont autant d'indicateurs de bien-être physique, mental et social. La place d'un individu dans la société affecte donc directement ce bien-être. Une femme travaillant à temps partiel à la caisse d'un grand magasin et élevant seule ses trois enfants a de fortes chances de voir baisser son niveau de bien-être physique, mental et social. Les résultats de la triple association de sa situation économique, professionnelle et personnelle doivent-ils faire l'objet d'un traitement médical ou bien politique et social ? En prenant pour point de départ la santé et non plus la maladie, ne prenons-nous pas le risque ici d'étendre si largement le filet de cette notion qu'il en vienne à recouvrir tout ce qui en était exclu jusqu'alors, à commencer par le registre socio-économico-politique ? Les SDF (sans domicile fixe) sont-ils dans la rue parce qu'ils présentent pour une forte partie d'entre eux des troubles mentaux, ou bien l'exclusion socio-économico-politique qui les a conduit dans la rue a-t-elle eu pour effet de déclencher chez eux des troubles mentaux consécutifs à un tel mode de vie ? Personne n'est aujourd'hui en mesure de répondre à cette question car elle illustre d'une part le principe de causalité circulaire qui est à l'œuvre au sein de nos interactions sociales et culturelles. D'autre part, elle nous révèle la porosité qui affecte les frontières que nos sociétés s'efforcent de maintenir entre santé et maladie, registre médical et registre économique, culturel et politique, et nous montre que toute Thérapeutique est 
Politique et inversement. L'espace du soin est un espace politique au sens où il exprime les normes, les principes et les valeurs qui animent ce dernier.

La psychiatrie de secteur en projetant le malade mental au cœur de la Cité ne dit pas autre chose : en passant d'une logique institutionnelle à une logique politique, la notion de santé mentale force nos sociétés à s'adapter aux malades mentaux, à les penser comme une partie d'elles-mêmes. Mais il ne faut pas occulter une conséquence majeure qui est le tribut de ce changement de logique : le pouvoir médical passe ici sous les fourches caudines du pouvoir politique dont il devient de manière évidente l'une des manifestations essentielles. Même s'il n'en a jamais été autrement, comme le montre Michel Foucault tout au long de son œuvre, et plus particulièrement dans "Naissance de la Clinique" [20], l'absorption du champ médical dans le champ politique est ici totalement achevée et illustre magistralement cette médecine sociale analysée dans la théorie foucaldienne du bio-politique 9 .

\subsection{Bio-Politique et lutte contre la stigmatisation}

$\mathrm{Au}$ point où nous en sommes, nous voyons apparaître le paradoxe suivant : selon notre lecture en effet, la psychiatrie de secteur - nécessairement alliée à la lutte contre la stigmatisation - constituent l'une des formes sophistiquées de l'exercice du bio-pouvoir. La fermeture des structures asilaires et la fin de l'enfermement des malades mentaux ne signifie pas que le périmètre de sécurité établi autour des pathologies mentales ait disparu, mais simplement qu'il s'est transformé et que l'espace du contrôle et de la régulation s'est considérablement élargi. Ce n'est plus la seule catégorie des malades mentaux qui fait l'objet de cette surveillance, mais la population tout entière non seulement en tant qu'elle accueille en son sein les malades mentaux, mais aussi en tant qu'elle fait elle-même l'objet d'un contrôle approfondi dont les enquêtes telles que "La santé mentale en population générale : Images et réalités", menée sous la responsabilité de l'Organisation mondiale de la Santé, constituent l'un des instruments les plus efficaces. Repérer, détecter, soigner, deviennent autant de gestes politiques qui échappent au strict registre médical et transforment les spécialistes de la santé mentale en auxiliaires précieux du bio-pouvoir.

\footnotetext{
${ }^{9}$ Pour Foucault, les modes de prise en charge du corps, de définition de la santé et de la maladie relèvent à partir du XVIII ${ }^{\circ}$ siècle d'une gestion étatique qui vise à étendre son pouvoir sur la vie des populations et des individus qui les constituent. Le développement de l'hygiène publique, de la médecine, l'optimisation des conditions de vie, de l'habitat, le recensement...participent de ce projet politique de gouvernance des esprits et des corps fondé sur le contrôle et la régulation. cf. [21].
} 
Le principe du dépistage anonyme, gratuit, et non présenté comme tel, les campagnes d'information et de prévention adressées à différents types de publics rendent inutiles les anciens processus de séparation et d'exclusion. On remarquera ici la grande proximité de cette démarche avec celle qui concerne les malades du sida. Les malades mentaux sortent de la juridiction médicale pour entrer de plain-pied dans la juridiction politique : dès lors, chacun devient potentiellement susceptible de faire l'objet d'un repérage/dépistage d'autant plus complet qu'il devient général. Ce nouvel agencement du pouvoir médical, tout entier au service du pouvoir politique, constitue ce que Foucault appelle un "dispositif de sécurité"10 [22] qui encourage les individus à modifier massivement leurs représentations et pratiques, la particularité de ces dispositifs de sécurité étant qu'ils cherchent moins à s'exercer sur les corps individuels que sur les populations. L'auteur analyse les objectifs de ces dispositifs de sécurité, analyse qui prend tout son sens dans le cas de la santé mentale. Il s'agit, dit-il, d'évaluer des taux qu'il conviendra de faire baisser ; d'enquêter non sur des individus mais sur des populations et des sous-populations en cherchant à observer des prévalences supérieures à la moyenne ; de veiller à pointer les dangers ; de ne pas interdire ni prescrire des comportements et des pratiques mais de chercher à s'appuyer sur elles pour développer la sensibilisation ; de mettre au point des campagnes d'information et de communication qui s'adressent à toute la population et non à des groupes discriminés.

Dès lors, la déstigmatisation des malades mentaux devient la clef de voûte de cet élargissement du "contrôle psy" à la population tout entière. L'efficacité d'une planification mondialisée du programme de santé mentale dépend de la réussite des campagnes de lutte contre la stigmatisation : chacun et tous doivent pouvoir se penser comme de futurs patients potentiels qu'un accident de la vie, une absence de structuration sociale, pourront conduire à requérir des soins psychiatriques autrefois réservés à un groupe d'individus isolés du corps social, groupes sur lesquels pesait le fatum biologique d'une pathologie sélective.

\subsection{Une économie bio-politique de la déstigmatisation}

De telle sorte que le dispositif politique et social de stigmatisation de la stigmatisation que représente le processus de déstigmatisation vient d'une part se loger à l'endroit même où les

\footnotetext{
${ }^{10}$ Cf. [22], page 61.
} 
stigmatisations classiques opéraient. D'autre part, nous permettre de faire l'économie de toutes les procédures de contention, d'exclusion, de séparation, de mise à l'écart qui coûtaient si cher jusque-là à nos sociétés et empêchaient le bio-pouvoir d'étendre son contrôle sur la totalité des populations qui les composent. Pour que la déstigmatisation des malades mentaux soit opérationnelle, il faut donc réunir un certain nombre de conditions.

Ainsi, la grande majorité des rapports spécialisés (produits en France et à l'étranger) concernant la stigmatisation des malades mentaux et la nécessité de réduire les discriminations auxquelles elle donne lieu, met en avant une raison fondamentale d'organiser la lutte contre ces dernières: la stigmatisation des malades mentaux retarde l'administration des soins et la prise en charge thérapeutique rapide des malades.

La déstigmatisation influence donc directement l'économie du soin et sa finalité. Elle possède une rentabilité économique et thérapeutique supérieure à la stigmatisation. C'est donc la première raison de la mobilisation bio-politique en sa faveur. Autrement dit la lutte contre la stigmatisation vient ici prendre la place de la stigmatisation dont le but visait autrefois, comme nous l'avons montré dans la première partie de cette étude, à protéger la société, ainsi qu'à augmenter et à prolonger la normalisation sociale. L'ironie du sort veut donc que la lutte contre la stigmatisation apparaisse aujourd'hui comme un instrument privilégié du redéploiement du pouvoir de contrôle socio-médical que dénonçaient précisément les critiques radicales des années 70, et tout particulièrement Michel Foucault, ce même Michel Foucault dont les théories sont aujourd'hui partout ou presque mobilisées contre lui-même, au service d'une cause dont il démontrerait sans doute la profonde ambivalence. La lutte contre la stigmatisation met en scène ces mécanismes de médiation médicale croissante des interactions sociales que dénonçait déjà Michel Foucault et qu'il attribuait tout particulièrement à l'institution de la fonction psy chargée de généraliser et de multiplier les modes de contrôle sur les individus et les groupes.

Comme nous pouvons le voir, le processus de déstigmatisation est le corollaire de l'externalisation de la fonction psy qui a migré du registre médical vers le registre politique et médiatique. En même temps qu'ils perdaient la garde des fous, les psychiatres ont perdu leur pouvoir spécifique et l'ont délégué au pouvoir politique. Ce double pouvoir politicomédiatique assigne aux psychiatres la lourde tâche de continuer à assumer la responsabilité 
des malades mentaux dans un espace ouvert, étendu à la totalité de la société. Leur rôle est d'entrer dans l'arène médiatique au moment où le système dysfonctionne : quand un malade mental commet un crime. L'exigence du pouvoir politique et médiatique exprime alors la même urgence : identifier le psychiatre et son équipe qui n'ont pas su prévenir et anticiper le geste criminel. Ce faisant, l'ordre politique et l'ordre médiatique récupèrent ainsi une deuxième fois la fonction psy. L'un et l'autre martèle que les psychiatres sont incompétents et que la folie est une chose trop sérieuse pour la confier à leurs seuls soins, fussent-ils spécialisés. Il faut s'interroger ici sur le rôle de la violence interrogée dans un tel cadre : elle justifie et renforce la migration de la fonction psy vers le politique puisque dans un système dominé par le couple médiatico-politique, chaque acte de violence renvoie le malade mental à la fatalité de sa maladie, et le psychiatre à l'aveu de son impuissance face à elle.

En ce sens, le double jeu des médias qui consiste à montrer pour informer et éduquer d'un côté, puis à surinvestir le sensationnel et l'émotionnel de l'autre, montre assez la profondeur de ce marché de dupes. La médiatisation outrancière de cette violence et sa récupération populiste semblent servir un projet politique dans lequel la fonction du boucémissaire (le fou, le médecin, l'hôpital hors les murs) et sa dimension expiatoire restent fichées au centre de nos sociétés, comme n'a cessé de le montrer le philosophe René Girard. Une violence fondatrice qui appelle à son tour la stigmatisation de ses auteurs et organisateurs dans un mouvement sans fin qui est celui même de nos cultures et fait signe une fois de plus vers l'ambivalence récurrente de la double demande socio-politique et médicale de la lutte contre la stigmatisation des troubles mentaux. Une ambivalence qu'il nous faut désormais penser moins comme une étape du processus de déstigmatisation que comme l'affirmation de la nature structurante et socialisante de la stigmatisation. Un peu comme si stigmatiser la stigmatisation revenait à jouer indéfiniment son jeu, pris au piège que nous sommes de ses mouvantes et multiples réincarnations dans le corps social. De cette dimension tragique de la stigmatisation nous pouvons dire ce que le dramaturge grec Sophocle disait d'Édipe-Roi : elle accomplit cela même qu'elle voulait empêcher. 


\section{Références}

[1] Bertini, Marie-Joseph, Femmes. Le pouvoir impossible, Paris, Pauvert / Fayard, 2002.

[2] Blin, Thierry, Phénoménologie de l'action sociale : A partir d'Alfred Schütz, Paris, L'Harmattan, 2000.

[3] Jodelet, Denise, Les représentations sociales, Paris, PUF, 1999.

[4] Searle, John, La construction de la réalité sociale, Paris, Gallimard, 1998.

[5] Bertini, Marie-Joseph, "Figures de la domination : les implications épistémiques et politiques de l'approche sophistique", Revue Recherches en Communication, Université de Louvain-la-Neuve, ${ }^{\circ} 24,2005$, pp. 196-205.

[6] Moscovici, Serge, La psychanalyse, son image et son public, Paris, PUF, 2004.

[7] Bourdieu, Pierre, Le sens pratique, Paris, éditions de Minuit, 1980.

[8] Bourdieu, Pierre, La domination masculine, Paris, Le Seuil, 1998.

[9] Bourdieu, Pierre, La distinction. Critique sociale du jugement, Paris, éditions de Minuit, 1979.

[10] Bourdieu, Pierre, Ce que parler veut dire. L'économie des échanges linguistiques, Paris,

Fayard, 1982.

[11] Bourdieu, Pierre, Langage et pouvoir symbolique, Paris, Le Seuil, 2001.

[12] Machado de Assis, Joachim-Maria, L'Aliéniste, Paris, Editions Métailié, 2005.

[13] Goffman, Erving, Stigmate. Les usages sociaux des handicaps, Paris, éditions de Minuit, 1975.

[14] Bougnoux, Daniel, Sciences de l'information et de la communication, Paris, Larousse, 1993.

[15] Maupassant de, Guy, Le Horla, Paris, Gallimard, 2003.

[16] Blanchot, Maurice, Faux Pas, Paris, Gallimard, 1943.

[17] Simmel, Georges, Le Pont et la Porte, in La tragédie de la culture et autres essais, Paris, Rivages, 1993.

[18] Girard, René, La violence et le sacré, Paris, Grasset, 1972. 
[19] Piel, Eric, et Roelandt, Jean-Luc, Rapport de mission au Ministère de l'emploi et de la solidarité, De la psychiatrie vers la santé mentale, Paris, La Documentation Française, 2001.

[20] Foucault, Michel, Naissance de la Clinique, Paris, PUF, 2009.

[21] Foucault, Michel, Naissance de la biopolitique. Cours au Collège de France (19781979), Paris, Gallimard /Editions du Seuil, 2004.

[22] Foucault, Michel, Sécurité, territoire, population. Cours au Collège de France (19771978), Paris, Editions du Seuil, 2004, page 61. 